\title{
A SMARTPHONE-BASED MULTI-SENSOR WIRELESS PLATFORM FOR CYCLING PERFORMANCE MONITORING
}

\author{
DIOGO S. OLIVEIRA \\ CMEMS-UMinho R\&D Center, University of Minho, Guimarães, \\ 4800-058,Portugal,e-mail: a52591@alunos.uminho.pt \\ JOSÉ A. AFONSO \\ CMEMS-UMinho R\&D Center, University of Minho, Guimarães, \\ 4800-058, Portugal, e-mail: jose.afonso@dei.uminho.pt
}

\begin{abstract}
In recent years there has been a significant evolution regarding applications for mobile devices that provide location-based services. The mobile devices available on the market already provide a set of integrated sensors and it is also possible to acquire data from external sensors. This chapter presents the development and results concerning a mobile sensing platform applied to cycling which performs data collection using both sensors integrated in the smartphone and multiple wireless sensor nodes, which are used to acquire relevant performance parameters. The data collected by the developed mobile app is stored in a local database and also uploaded to a remote database, where it can be accessed later using the mobile app or a web browser. This mobile app allows users to share data with friends, join or create events, locate friends, consult graphs and access past routes in a map. Based on these functionalities, this system aims to provide detailed feedback regarding the user performance and enhance the enjoyment of the cyclists.
\end{abstract}

\section{Introduction}

Smartphones are increasingly becoming the central communication and processing devices in people's lives, which makes the study and development of mobile sensing systems an attractive emerging area of research [1], [2].

In order to enhance their performance and enjoyment, cyclists are increasingly interested in multiple types of information that can be gathered. The use of smartphones in cycling has the advantages of avoiding the costs associated with the acquisition of dedicated monitoring devices and, at the same time, allowing the provision of several new functionalities.

Depending on the intended goals, cycling exercises at specific intensity zones may be advisable. These zones are usually calculated based on the measurement of the power output or the heart rate [3], [4]. The cycling performance is also largely affected by the body posture [5]. There are performance tradeoffs between the upright posture and the aero posture (where the cyclist adopts a lowered position with the trunk almost horizontal), which also depend on the 
fitness level of the cyclist [6]. The performance of cyclists is also affected by the angle of the knee [7]. Taking into account their relevance to the cycling performance, the system described in this chapter was designed to monitor parameters such as the pedaling cadence, torque, power output, heart rate, trunk angle and knee angle.

Among the research work applied to cycling, the Biketastic platform [8] aims to facilitate the exchange of knowledge among cyclists, creating a system where participants can share their routes and experiences. Data are collected through the sensors integrated into a smartphone. Through GPS, information such as speed, latitude and longitude is collected. Using the accelerometer and the microphone, it is possible to measure the roughness of the terrain and the level of audible noise. Users can also capture geo-tagged images and video clips while riding, and insert tags and descriptions.

BikeNet [9] is a monitoring system to map the experience of the cyclist, which uses a number of sensors to collect data on the cyclist routes and provides a web portal that allows sharing of information in real time and accessing the information stored on a server. This system collects and stores typical data, such as current speed, average speed, distance and calories burned, as well as more specific data, such as slope, heart rate, cadence, galvanic skin response, pollution levels, allergen levels, noise and roughness of the terrain, all referenced by time and location. The monitored data are collected by various IEEE 802.15.4 [10] sensor nodes, and an IEEE 802.15.4/Bluetooth gateway is used to send the data from the sensor nodes to a smartphone.

This chapter, which provides a revised and extended version of a previous paper [16], presents a system that allows the user to monitor several parameters during the cycling activities. Some of these parameters are monitored using smartphone sensors, whereas others use wireless sensors nodes [11] placed on the bicycle and on the user's body [12]. All information is georeferenced with the GPS location. The developed mobile app allows viewing, storing and sharing maps of the routes with friends, and the use of color gradients to provide visual information associated with changes on the monitored parameters. The mobile app also allows the user to be informed and join events where other cyclists will participate, and to create events himself. The data collected from all the users is stored on a remote database. Access to the monitored data and to the associated functionalities is available through the mobile app and through a website.

This chapter is organized as follows: The next section provides a description of the components of the developed system, with emphasis on the databases and the mobile app. In Section 3, experimental results concerning the measurement of specific parameters are presented and discussed. Finally, Section 4 presents the conclusions. 


\section{Developed System}

The architecture of the developed system, presented in Fig. 1, is composed of the main following components: a web server, a website, a mobile app and an external web service, which provides Google Maps geographic data.

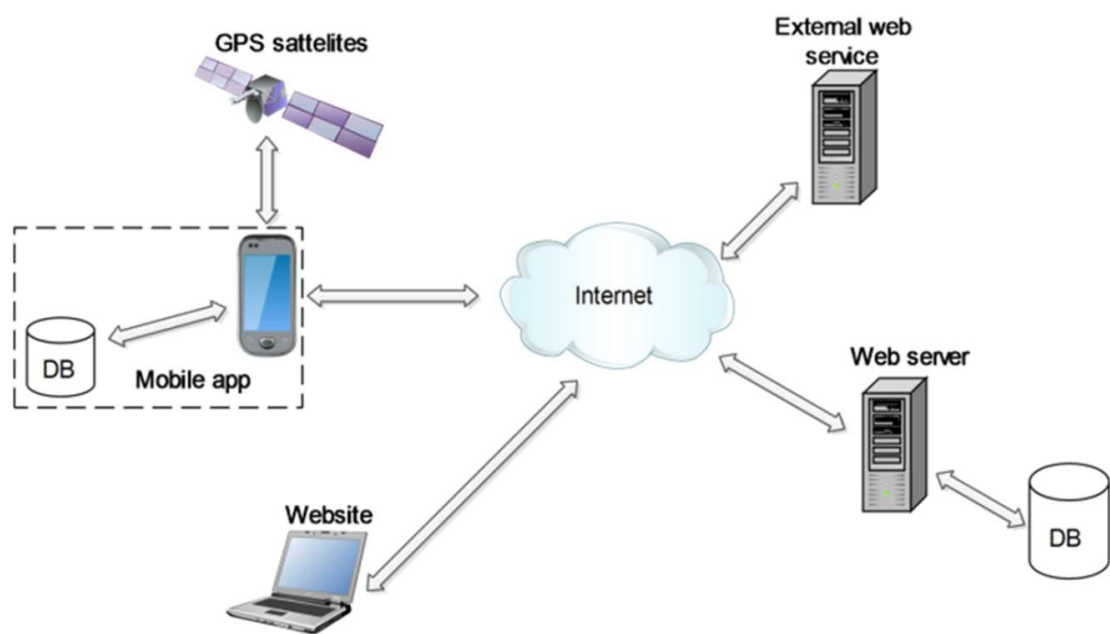

Fig. 1. Architecture of the developed system.

The web server handles requests made by the user, either through the smartphone application or a web browser. This web server integrates a database (DB) which stores information relevant to the operation of the system. The Apache HTTP server was chosen for the implementation of the web server, more specifically, the free XAMPP Apache distribution, because it contains MySQL and PHP. The databases are described in Section 2.1.

The mobile app possesses its own local database, which was developed using SQLite. It communicates with the web server in order to store collected information or to request information stored in the remote database. The mobile app, which was developed and tested using the Android mobile operating system, is described in more detail in Section 2.2, whereas the developed website is described in Section 2.3.

The communication between the mobile app and the web server is made using the HTTP POST method and the JSON (JavaScript Object Notation) format. The choice of the JSON format was due to the fact that it requires less bandwidth than the XML (Extensible Markup Language) format, contributing to the reduction of battery energy and mobile data consumption. 


\subsection{Databases}

The remote database, located on the web server, stores information collected from all the users of the system. This work uses the MySQL relational database management system (RDBMS). Fig. 2 presents the entity-relationship (ER) diagram that was developed for this database, which is composed of the following entities:

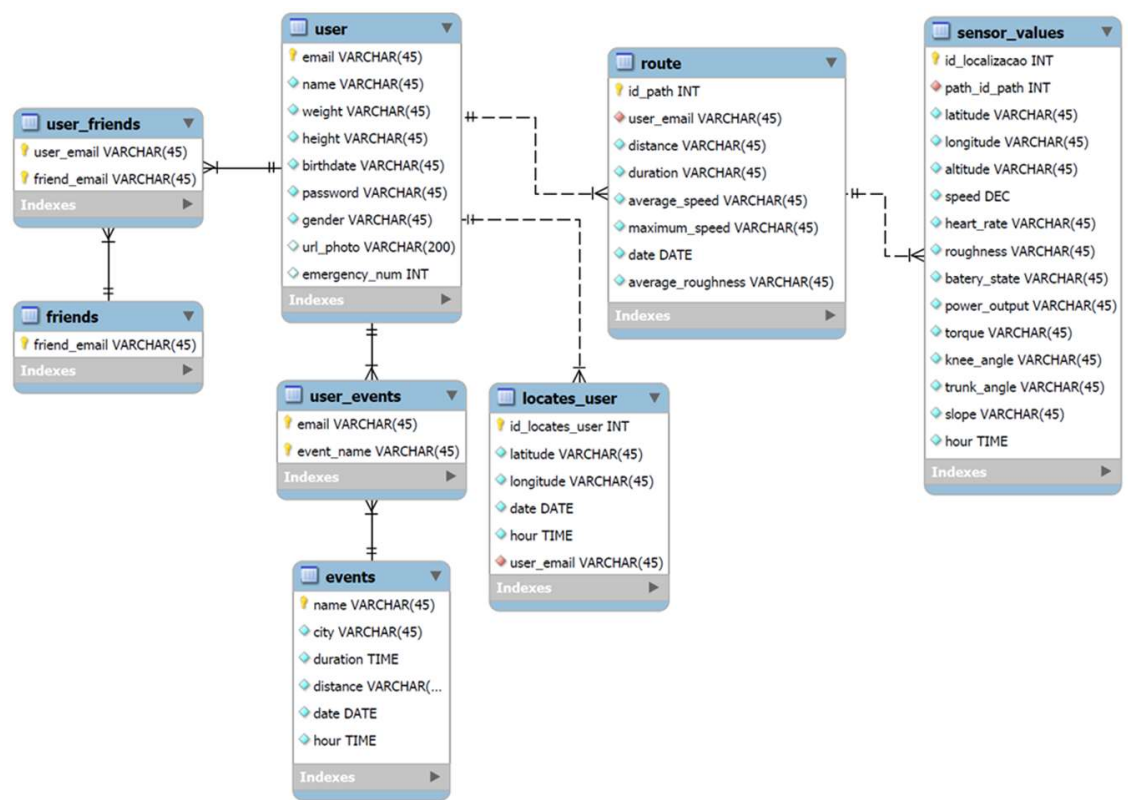

Fig. 2. Entity-relationship diagram of the remote database.

- user - stores information related to the user;

- locates user - contains information required to obtain the last location of the user;

- route - contains information related to each route performed by the user;

- sensor_values - contains information collected from a route;

- user events - contains information about the events that the user is associated to;

- events - stores information about the event;

- user_friends - contains information on which friends associated with the user;

- friends - stores the emails of friends, so that the user can later access information related to his friends.

The local database in the smartphone stores information regarding the routes performed by the user. The entities of this database are: the route, which 
contains the information on route performed by the user; and the sensor values, which contains all the data collected in the course of a route. These entities are very similar to the corresponding entities on the ER diagram of the web server; the idea is to facilitate the data upload from local database to the remote database.

\subsection{Mobile App}

The top level navigation on the mobile app is based on an Android spinner, with different options, as shown in Fig. 3. Each of these options opens another window that gives access to the respective sub-options.

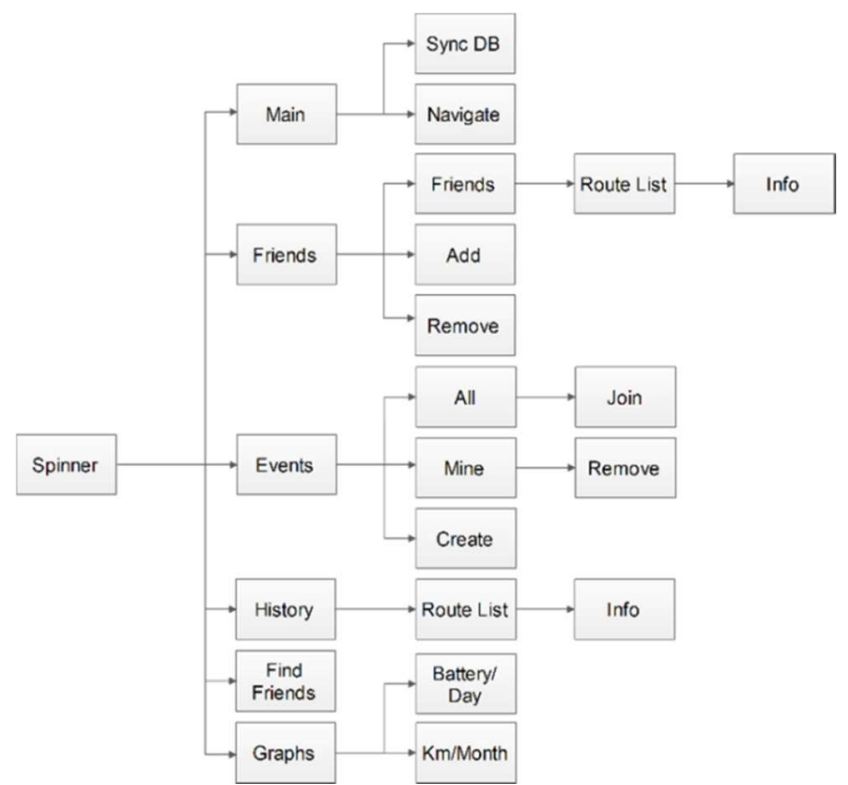

Fig. 3. Menu options available using the Android spinner.

The Main window allows the user to: sync the local database with the remote database manually (when $\mathrm{Wi}-\mathrm{Fi}$ is available, the data upload occurs automatically); or to start the navigation mode, which is the main part of the application.

The navigation window presents the local map and a start/stop button that allows the user to enable/disable the recording of the route information. When recording is enabled, the following georeferenced parameters are measured periodically, as long as the respective sensors are available:

- from smartphone sensors - roughness and slope of the terrain, speed, altitude and location; 
- from wireless body sensors - heart rate, knee angle and trunk angle;

- from wireless sensors placed on the bicycle - cadence, torque and power output.

This information is stored in the local database, to be later uploaded to the remote database.

As shown in Fig. 4, while on the navigation window, the user has access to the following information: elapsed time, current speed, distance traveled, altitude, and temperature, as well as the route traced on the map.

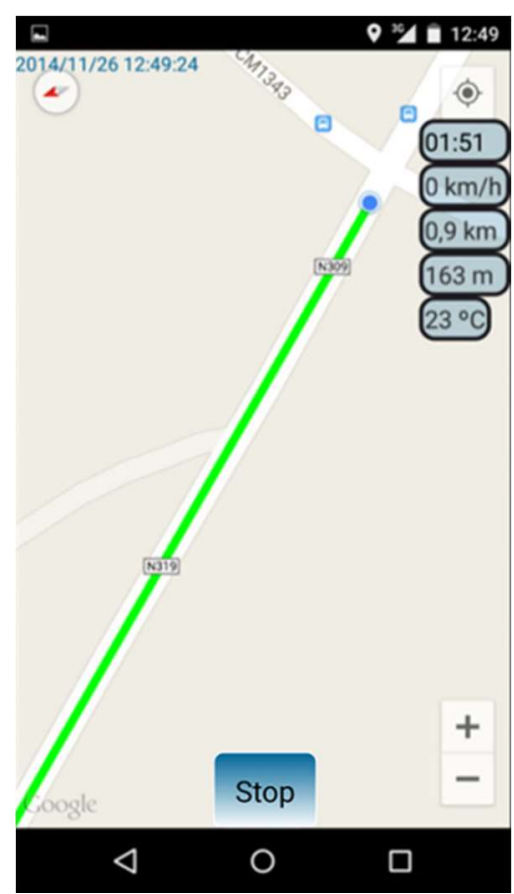

Fig. 4. Route recording on navigation mode

The option Friends presents to the user a swipe view with three tabs: Friends, Add and Remove. The tab Friends presents the friends of the user in a dynamic listview containing photo, name and email. Selecting a friend, the user has access to a listview with the history of routes performed by that friend. Selection of a route provides a summary, as well as access to a map where the route can be inspected. The tab Add provides two options to the user: give permission to another user to become a friend or ask another user to add him as a friend. The tab Remove allows the user to remove other users from the list of friends.

When the option Events is selected, it presents three tabs: All, Mine and Create. The tab All presents a list of future events that the user can join and 
respective information: name of the event, place, date, hour, estimated duration and distance. The tab Mine allows to consult or remove events that the user subscribed, whereas the tab Create allows the user to create a new event.

The option History presents a dynamic listview with all past routes performed by the user. Selecting a route provides more information and gives access to a map with the traced route.

Selecting the option Find Friends, the user has access to a map with markers that indicate the last known location of his friends and the respective time.

The option Graphs gives access to charts. Currently, two graphs are implemented: the smartphone battery level along the day, and the monthly kilometers travelled during the year. The graphs were implemented using the library AChartEngine.

\subsection{Website}

The website was developed using HTML, JavaScript and CSS, resorting to the Bootstrap framework. It was conceived to be suitable to multiple types of device, including PCs, tablets and smartphones. The Google Maps JavaScript API version 3 was used to incorporate maps on the website, whereas the Google Charts API was used to create charts using JavaScript embedded in the web page.

Fig. 5 presents main navigation options of the website. As the figure shows, the website allows the access to information from friends, events, past routes and charts.

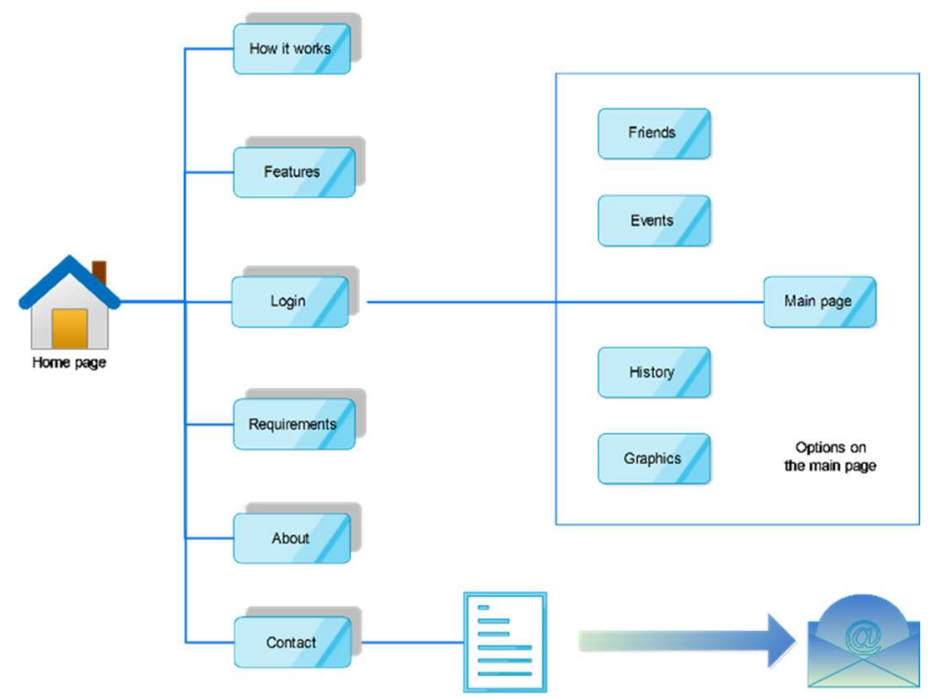

Fig. 5. General scheme of the website. 


\section{Results and Discussion}

This section presents experimental results concerning the measurement of several parameters using the developed system, namely the roughness of the terrain, cadence, torque, knee angle and trunk angle.

The roughness of the terrain (as well as the slope) is measured with the smartphone attached to the bicycle handlebars, as shown in Fig. 6.

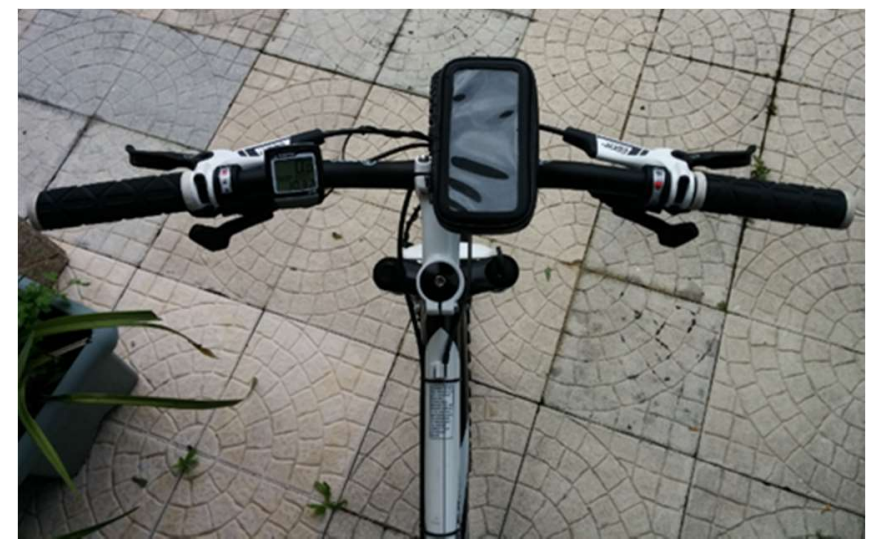

Fig. 6. Placement of the smartphone for the roughness test.

The Android SensorEvent class was used to measure the acceleration on the three axes $\left(a_{x}, a_{y}, a_{z}\right)$, using the accelerometer of the smartphone. Then the module of the acceleration was calculated, at 100-ms intervals, using (1). When the device is at rest, this equation returns the acceleration of gravity.

$$
|a|=\sqrt{a_{x}^{2}+a_{y}^{2}+a_{z}^{2}}
$$

Fig. 7 shows the results concerning the measurement of the module of the acceleration along the time for two different terrains: asphalt and brick road. After subtracting the acceleration of gravity from the module of the acceleration, the root mean square (RMS) of the samples was calculated for each terrain, in order to obtain a roughness factor for that terrain. The obtained value was 1.79 $\mathrm{m} / \mathrm{s}^{2}$ for the asphalt road and $6.93 \mathrm{~m} / \mathrm{s}^{2}$ for the brick road, which is a considerable difference.

The sharing of roughness data, in combination with other information, such as changes on the altitude along a route, may be useful for cyclists to choose or avoid routes according to the characteristics of the desired terrain.

In order to provide an easy visualization of changes on selected measured parameters along the route, the developed mobile app offers the option to display the route using a color gradient. Fig. 8 presents an example where the 
altitude is used. Darker red colors indicate higher altitudes along the route, whereas lighter colors indicate lower altitudes.

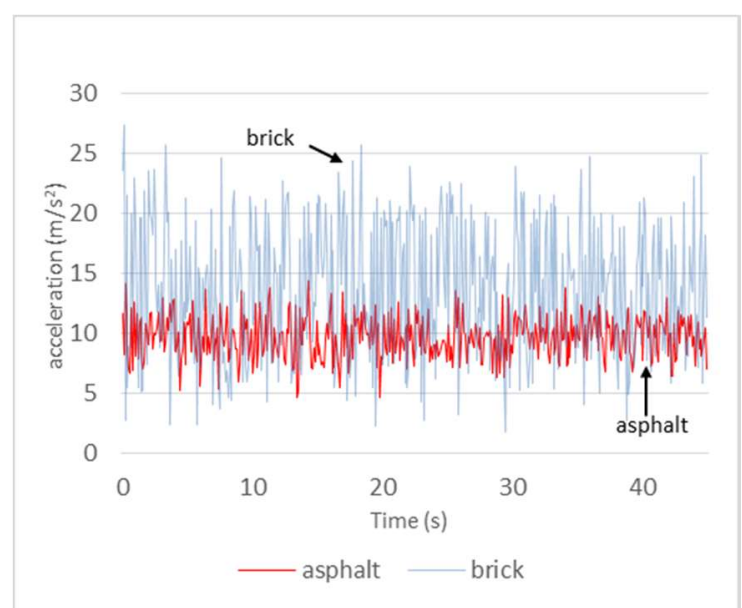

Fig. 7. Module of the acceleration over time for different terrains.

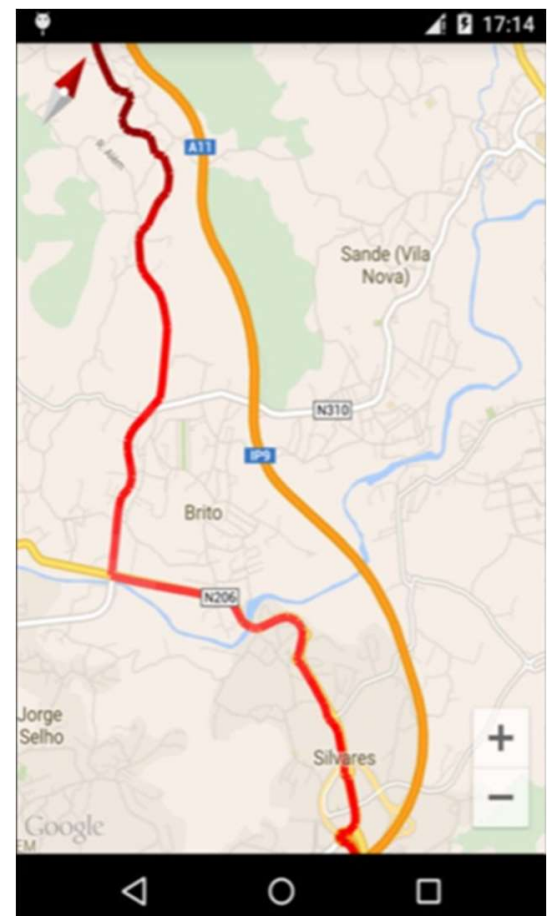

Fig. 8. Example of route using a color gradient for the altitude. 
In the system described in this work, the cadence, torque and power output are measured using a single sensor, which was placed inside of the bicycle crankset. This sensor provides an analog signal that is proportional to the torque applied by the cyclist, as well as a digital signal that is generated as the pedal is rotated ( 8 pulses per $360^{\circ}$ rotation). These signals are acquired by microcontroller and sent to the smartphone using Bluetooth. The value of the cadence, which is calculated on the mobile app, is inversely proportional to the time between two consecutive pulses of the digital signal. Fig. 9 shows an example of the measurement of the cadence along the time during a test.

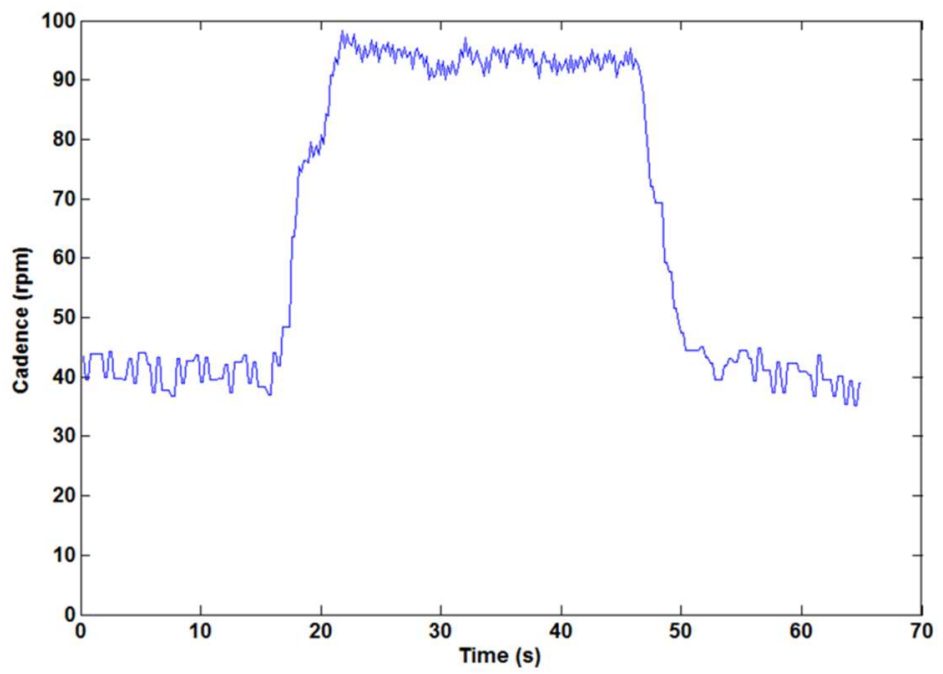

Fig. 9. Measured cadence based on the time between pulses generated by the sensor placed inside of the bicycle crankset.

Fig. 10 presents an example of the torque measured during another test. As the figure shows, the magnitude of the signal provided by the used sensor changes considerably as the cyclist presses and releases the pedal. Therefore, before the torque value is stored on the database, it is averaged through the application of the EWMA (Exponentially Weighted Moving Average) technique. The power output, in $\mathrm{W}$, is readily obtained through the multiplication of the torque, in $\mathrm{Nm}$, by the cadence, in $\mathrm{rad} / \mathrm{s}$. Its value is also averaged before it is stored. In the current prototype, the heart rate is collected using a Zephyr HxM Bluetooth monitor.

The body angles are measured using sensor modules [13] that integrate 3-axis accelerometers and magnetometers. One module is used to measure the trunk angle and two are required to measure the knee angle [14]. The data generated by the modules is sent to the smartphone using Bluetooth Low Energy (BLE) 
[15]. Fig. 11 presents an example of cycling activity where the cyclist is adopting an upright position (trunk angle is near zero) when he starts pedaling. At $26 \mathrm{~s}$, the cyclist starts bending forward, adopting a more aerodynamic position (trunk angle increases), and also increases the pedaling cadence, which is reflected on a faster variation of the knee angle.

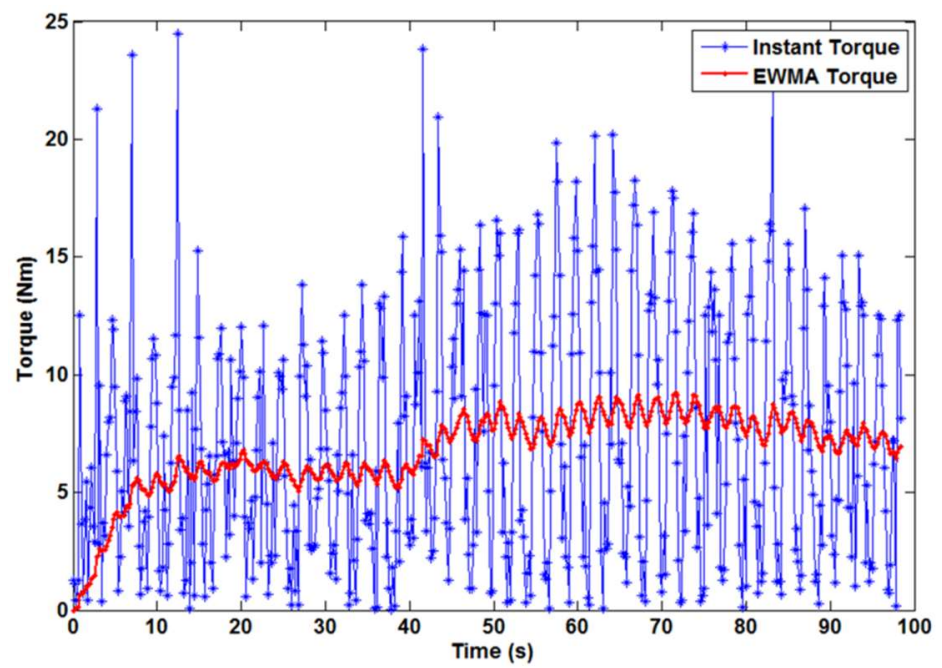

Fig. 10. Measured torque before and after the application of the EWMA technique.

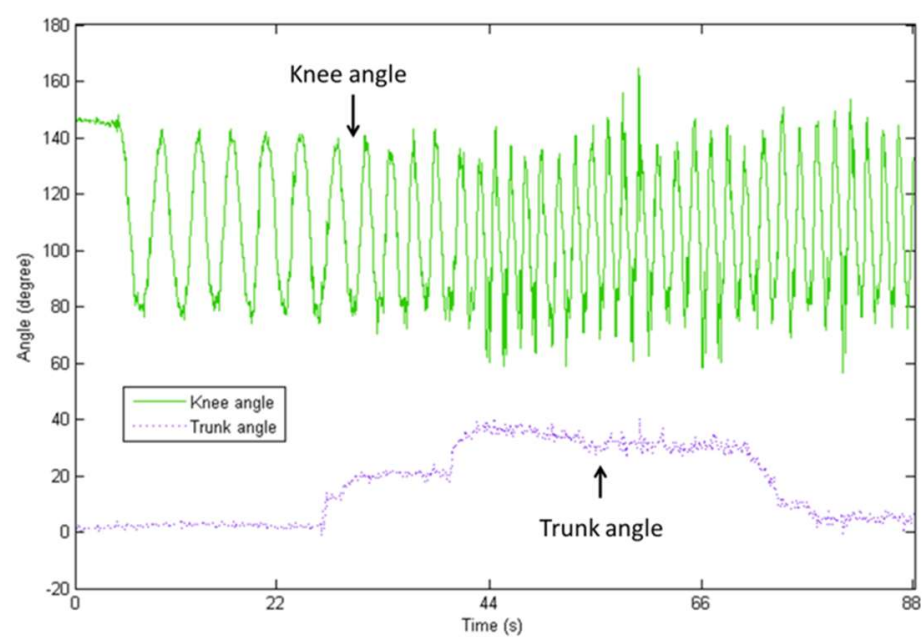

Fig. 11. Knee and trunk angles measured during a cycling activity. 


\section{Conclusions and Future Work}

Besides the typical parameters monitored by available smartphone applications, such as speed and distance, the system presented in this chapter allows the monitoring of several performance parameters associated to the exercise intensity and body posture, as well as data associated to the characteristics of the cycling trails. Together with the functionalities of sharing the routes with friends, creating and joining events, and accessing routes and charts, this system aims to contribute to increase the performance feedback at lower costs and to enhance the user's experience.

In the current prototype, some sensors nodes send data to the smartphone using Bluetooth, while others use BLE. In the future we intend to migrate all sensors nodes to BLE, because BLE presents lower energy consumption than Bluetooth, in order to use a single wireless interface to the sensors nodes on the smartphone.

The perspectives for future work include also the development and test of methods for automatic recognition and classification of the type of surface (e.g., asphalt, crushed stone, gravel or grass) based on the acceleration patterns measured using the smartphone sensors.

In the future we also intend to integrate this mobile app with another developed Android app, which provides several other personalized functionalities for cyclists, such as an interface to public transportation information [17].

When used with electric bicycles (EBs), the system described in this chapter may also be enhanced with functionalities such as EB range prediction [17] or automatic control of the effort exerted by the cyclist [18], using some of the sensors presented in this work. In this case, the controlled effort parameter may be selected by the cyclist between three different options: the pedaling resistance (based on the measured torque), the power output (based on the torque and pedaling cadence) or the heart rate.

\section{Acknowledgments}

This work has been supported by FCT (Fundação para a Ciência e Tecnologia) in the scope of the project: UID/EEA/04436/2013.

\section{References}

1. N. D. Lane, E. Miluzzo, L. Hong, D. Peebles, T. Choudhury, and A. T. Campbell, "A survey of mobile phone sensing," IEEE Communications Magazine, 48(9), 2010, pp140-150. 
2. W. Z. Khan, Y. Xiang, M. Y. Aalsalem, and Q. Arshad, "Mobile phone sensing: A survey," IEEE Communications Surveys \& Tutorials, 15(1), 2013, pp402-427.

3. G. A. Pussieldi, C. E. Veneroso, K. M. C. Pinto, G. M. Bresciani, M. M. Teixeira and J. A. Fernández., "Effort control through heart rate in an off road cross country bicycle race," Fitness \& Performance Journal, no. 5, 2005, pp299-305.

4. F. Impellizzeri, A. Sassi, M. Rodriguez-Alonso, P. Mognoni, and S. Marcora, "Exercise intensity during off-road cycling competitions," Medicine and Science in Sports and Exercises, 34(11), 2002, pp1808-1813.

5. M. Egaña, S. Green, E. J. Garrigan and S. Warmington, "Effect of posture on high-intensity constant-load cycling performance in men and women," European Journal of Applied Physiology, 96(1), 2003, pp1-9.

6. M. C. Ashe, G, C, Scroop, P, I, Frisken, C, A, Amery, M, A, Wilkins and K. M. Khan, "Body position affects performance in untrained cyclists," British Journal of Sports Medicine, 37(5), 2003, pp441-444.

7. W. Peveler, J. Pouders and P. Bishop, "Effects of saddle height on anaerobic power production in cycling," Journal of Strenght and Conditioning Research, 21(4), 2007, pp1023-1027.

8. S. Reddy, K. Shilton, G. Denisov, C. Cenizal, D. Estrin, M. Srivastava "Biketastic: Sensing and Mapping for Better Biking," CHI 2010: Bikes and Buses, New York, USA, April 2010, pp1817-1820.

9. S. Eisenman, E. Miluzzo, N. Lane, R. Peterson, G. Ahn, and A. Campbell, "The BikeNet mobile sensing system for cyclist experience mapping," 5th International Conference on Embedded Networked Sensor Systems, Sydney, Australia, 2007, pp87-101.

10. IEEE Std 802.15.4-2006, "IEEE standard for local and metropolitan area networks - Part 15.4: Wireless medium access control (MAC) and physical layer (PHY) specifications for low-rate wireless personal area networks (WPANs)," September 2006.

11. J. Yick, B. Mukherjee, and D. Ghosal, "Wireless sensor network survey," Computer Networks, 52(12), 2008, pp2292-2330.

12. M. Chen, S. Gonzalez, A.Vasilakos, H. Cao, and V. C. M. Leung, "Body area networks: A survey," Mobile Networks and Applications, 16(2), 2011, pp171-193.

13. J. A. Afonso, J. H. Correia, H. R. Silva, L. A. Rocha, "Body Kinetics Monitoring System," International Patent WO/2008/018810A2, February 2008.

14. A. Maio and J. A. Afonso, "Wireless Cycling Posture Monitoring Based on Smartphones and Bluetooth Low Energy," Lecture Notes in Engineering and Computer Science: Proceedings of The World Congress on Engineering 2015, WCE 2015, 1-3 July, 2015, London, U.K., pp 653-657. 
15. C. Gomez, J. Oller and J.Paradells, "Overview and evaluation of Bluetooth low energy: an emerging low-power wireless technology," Sensors, vol. 12, no. 9, 2012, pp. 11734-11753.

16. Diogo S. Oliveira and J. A. Afonso, "Mobile Sensing System for Georeferenced Performance Monitoring in Cycling," Lecture Notes in Engineering and Computer Science: Proceedings of The World Congress on Engineering 2015, WCE 2015, 1-3 July, 2015, London, U.K., pp 269-273.

17. João C. Ferreira, Vítor Monteiro, José A. Afonso and João L. Afonso, "Mobile Cockpit System for Enhanced Electric Bicycle Use", IEEE Transactions on Industrial Informatics, 11(5), October 2015, pp 1017-1027.

18. José A. Afonso, Filipe J. Rodrigues, Delfim Pedrosa and João L. Afonso, "Automatic Control of Cycling Effort Using Electric Bicycles and Mobile Devices," Lecture Notes in Engineering and Computer Science: Proceedings of The World Congress on Engineering 2015, WCE 2015, 1-3 July, 2015, London, U.K., pp 381-386. 